\title{
EEG SOURCE LOCALIZATION BY MULTI-PLANAR ANALYTIC SENSING
}

\author{
D. Kandaswamy ${ }^{1}$, T. Blu ${ }^{2}$, L. Spinelli ${ }^{3}$, C. Michel ${ }^{3}$ and D. Van De Ville ${ }^{1}$ \\ ${ }^{1}$ Biomedical Imaging Group, École Polytechnique Fédérale de Lausanne, CH-1015 Lausanne \\ 2 Department of Electronic Engineering, Chinese University of Hong Kong, Shatin N.T. Hong Kong \\ ${ }^{3}$ Functional Brain Mapping Group, University of Geneva, CH-1211 Geneva
}

\begin{abstract}
Source localization from EEG surface measurements is an important problem in neuro-imaging. We propose a new mathematical framework to estimate the parameters of a multidipole source model. To that aim, we perform 2-D analytic sensing in multiple planes. The estimation of the projection on each plane of the dipoles' positions, which is a non-linear problem, is reduced to polynomial root finding. The 3-D information is then recovered as a special case of tomographic reconstruction. The feasibility of the proposed approach is shown for both synthetic and experimental data.
\end{abstract}

Index Terms - EEG, source localization, finite rate of innovation, annihilating filter, dipole models, analytic functions

\section{INTRODUCTION}

Imaging the functioning of the human brain is an important task in neurosciences and neurology. To access the temporal properties of the brain circuits, electro- and magnetoencephalography (EEG, MEG) are predominant since they allow measuring signals down to millisecond resolution. Mapping back the measured signal $\left.V\right|_{\partial \Omega}$ on the scalp surface $\partial \Omega$ to the source configuration $\rho$ inside the brain is known as "source imaging" $[1,4]$. Unfortunately, the underlying electromagnetic inverse problem is ill posed; i.e., an infinity of different source configurations can explain the same scalp potential. Therefore, additional assumptions are required to make the solution unique. For this purpose, the various methods available are putting forward different hypotheses about the source model (single-dipole, multi-dipole, or distributed) and its properties (e.g., smoothness).

We propose a new theoretical framework that leads to a non-iterative technique for EEG multi-dipole fitting. We designate our method by "analytic sensing", since the key contribution is to apply analytic sensors (functions $\psi$ with vanishing Laplacian in some domain) that sense the influence of the source distribution $\rho$ in a specific region. Next, the non-linear

This work was supported by the Swiss National Science Foundation under Grant 200021-119812 and by the Center for Biomedical Imaging of the Geneva-Lausanne Universities and the EPFL, as well as the foundations Leenaards and Louis-Jeantet estimation problem associated to the dipole positions is performed by rephrasing the problem within the framework of "finite rate of innovation" [8]. Finally, the dipolar moments are retrieved by solving a linear system of equations. We extend the 2-D approach, which has been presented before [2], to 3-D by applying it to multiple planes. Each 2-D localization provides us with the projection of the dipoles' positions and moments onto the respective plane.

The paper is organized as follows. In Sect. 2, we introduce the problem setting. Next, in Sect. 3, we summarize the 2-D analytic sensing approach and its multi-planar extension. Finally, we demonstrate the feasibility of the framework by localizing at the early instant of visual evoked response potentials (ERPs). For this case, state-of-the-art localization using a distributed source model renders a well localized brain area. Multi-planar analytic sensing gives consistent results and leads to 3-D information that is in accordance to the standard algorithms.

\section{PROBLEM FORMULATION}

In EEG, one measures the electrical potential $V$ on the scalp surface $\partial \Omega$. These potential differences are the manifestation of a source distribution $\rho$ in the brain, which is governed by the equation:

$$
-\nabla \cdot(\sigma \nabla V)=\rho,
$$

where $\sigma$ is the conductivity of the head. A popular head model is the multishell spherical head model with piecewise constant conductivities [5]. Since no current can leave the head, we also have the following boundary condition:

$$
\left.(\nabla V)\right|_{\partial \Omega} \cdot \mathbf{e}_{\Omega}=0,
$$

where $\mathbf{e}_{\Omega}$ is the outward surface normal. The problem at hand, which is the inverse problem associated to EEG, can be described as: from $\left.V\right|_{\partial \Omega}$, reconstruct $\rho$.

However, determining $\rho$ is an ill-posed problem as the solution is non-unique. Here, we follow the approach to restrict $\rho$ to a parametric source model. Specifically, the multi-dipole model can be written as

$$
\rho(\mathbf{x})=\sum_{k=1}^{K} \mathbf{p}_{k} \cdot \nabla \delta\left(\mathbf{x}-\mathbf{x}_{k}\right),
$$


where we have $6 K$ unknowns; i.e., the dipoles' positions $\mathbf{x}_{k}=\left[\begin{array}{lll}x_{k} & y_{k} & z_{k}\end{array}\right]^{T}$ and moments $\mathbf{p}_{k}=\left[\begin{array}{lll}p_{k, 1} & p_{k, 2} & p_{k, 3}\end{array}\right]^{T}$, $k=1, \ldots, K$.

\section{MULTI-PLANAR ANALYTIC SENSING}

\subsection{Source Localization in the Planar Domain}

Analytic Sensing: We consider a closed 2-D region $\Omega$ with homogeneous conductivity $\sigma=1$. Then, the basic Laplace equation, $-\Delta V=\rho$, holds within $\Omega$. Applying Green's theorem yields the following line integral:

$$
\langle\psi, \rho\rangle=-\oint_{\partial \Omega} V \nabla \psi \cdot \mathbf{e}_{\Omega} d s
$$

where we have taken into account the boundary condition (2) and $\left.\Delta \psi\right|_{\Omega}=0$. Hence, using a set of analytic sensors, we can compute the generalized measures containing information on $\rho$ knowing only $\left.V\right|_{\partial \Omega}$. Let us now consider a specific set of analytic sensors:

$$
\psi_{a_{n}}(x, y)=\ln \left(x+i y-a_{n}\right)
$$

where $a_{n}=\alpha e^{i n \theta}, \alpha \in \mathbb{R}^{+}, n=0, \ldots, N-1$, such that $a_{n} \notin \Omega$. Since we need at least $2 K$ distinct measures, we choose $N \geq 2 K, \theta \leq \frac{2 \pi}{N}$.

Annihilation: The main principle behind our technique is that the dipole positions $\mathbf{x}_{k}=\left[\begin{array}{ll}x_{k} & y_{k}\end{array}\right]^{T}$ can be found as the complex roots $x_{k}+i y_{k}$ of a polynomial of degree $K$. Under the assumption of the source model, the generalized measures should also equal

$$
\left\langle\psi_{a_{n}}, \rho\right\rangle=\sum_{k=1}^{K} \mathbf{p}_{k} \cdot \nabla \psi_{a_{n}}\left(\mathbf{x}_{k}\right),
$$

which can be rephrased under the form $\sum_{k=0}^{K-1} c_{k}^{\prime} e^{i n k \theta} / R\left(a_{n}\right)$, $c_{k}^{\prime} \in \mathbb{R}$, with $R(X)=\sum_{k=0}^{K} r_{k} X^{k}$ a polynomial of degree $K$ with roots $x_{k}+i y_{k}$. The key observation is that the numerator $\sum_{k=1}^{K-1} c_{m}^{\prime} e^{i n k \theta}$ can be annihilated by a filter $h$ with $\mathcal{Z}$-transform

$$
H(z)=\sum_{k \in \mathbb{Z}} h_{k} z^{-k}=\prod_{k=0}^{K-1}\left(1-e^{i k \theta} z^{-1}\right) .
$$

Therefore, we have that

$$
h_{n} *\left(R\left(a_{n}\right)\left\langle\psi_{a_{n}}, \rho\right\rangle\right)=0
$$

holds, which is a linear system of equations in $r_{k}$. Solving (8) determines the polynomial $R$, and the dipole positions from its roots. The dipole moments $\mathbf{p}_{k}=\left[p_{k, 1} p_{k, 2}\right]^{T}$ are obtained by solving a linear system of equations (6) with known $\mathbf{x}_{k}$.

\subsection{Multi-planar Extension}

The generalized measures (4) can be readily extended to 3-D. Indeed, the line integral simply turns into a surface integral over $\partial \Omega$. Next, the same 2-D analytic sensing approach can be applied for a number of different planes. Specifically, we propose the analytic sensors

$$
\psi_{a_{n}}\left(x^{\prime}, y^{\prime}\right)=\ln \left(x^{\prime}+i y^{\prime}-a_{n}\right), \quad a_{n} \notin \Omega,
$$

in the (arbitrary) $\mathrm{X}^{\prime} \mathrm{Y}^{\prime}$-plane, which is defined by applying the coordinate transformation

$$
\begin{aligned}
& {\left[\begin{array}{l}
x^{\prime} \\
y^{\prime} \\
z^{\prime}
\end{array}\right]=} \underbrace{\left[\begin{array}{ccc}
1 & 0 & 0 \\
0 & \cos \theta & \sin \theta \\
0 & -\sin \theta & \cos \theta
\end{array}\right]}_{\mathbf{R}_{\theta}^{X}} \\
& \underbrace{\left[\begin{array}{ccc}
\cos \phi & 0 & \sin \phi \\
0 & 1 & 0 \\
-\sin \phi & 0 & \cos \phi
\end{array}\right]}_{\mathbf{R}_{\phi}^{Y}}\left[\begin{array}{l}
x \\
y \\
z
\end{array}\right]
\end{aligned}
$$

to the XY-plane. Each 2-D localization yields the projected dipole positions

$$
\underbrace{\left[\begin{array}{c}
x_{k}^{\prime} \\
y_{k}^{\prime}
\end{array}\right]}_{\mathbf{x}_{k, \theta, \phi}^{\prime}}=\underbrace{\left[\begin{array}{lll}
1 & 0 & 0 \\
0 & 1 & 0
\end{array}\right] \mathbf{R}_{\theta}^{X} \mathbf{R}_{\phi}^{Y}}_{\mathbf{A}_{\theta, \phi}}\left[\begin{array}{l}
x_{k} \\
y_{k} \\
z_{k}
\end{array}\right],
$$

and, accordingly, the projected moments. By considering a set of planes, characterized by the angles $\left\{\theta_{m}, \phi_{m}\right\}_{m=1, \ldots, M}$, the $3-\mathrm{D}$ reconstruction boils down to a special case of tomographic reconstruction. In the case where the projections of the dipoles can be easily separated, one can obtain the 3-D information by a least-squares fit

$$
\left[\begin{array}{c}
\hat{x}_{k} \\
\hat{y}_{k} \\
\hat{z}_{k}
\end{array}\right]=\left[\begin{array}{c}
\mathbf{A}_{\theta_{1}, \phi_{1}} \\
\vdots \\
\mathbf{A}_{\theta_{M}, \phi_{M}}
\end{array}\right]^{P}\left[\begin{array}{c}
\mathbf{x}_{k, \theta_{1}, \phi_{1}}^{\prime} \\
\vdots \\
\mathbf{x}_{k, \theta_{M}, \phi_{M}}^{\prime}
\end{array}\right],
$$

where ${ }^{P}$ designates the Moore-Penrose matrix inverse.

Here, we also use the multi-planar projections to evaluate the robustness of the analytic sensing approach. Ideally, the "sinogram" that is obtained by letting vary the planar angles should contain simple trigonometric combinations of $\theta$ and $\phi$ only.

\section{EXPERIMENTAL RESULTS}

In this section, we show the feasibility of the multi-planar analytic sensing technique with both synthetic and experimental data. We use a simple spherical head model with homogeneous conductivity. 


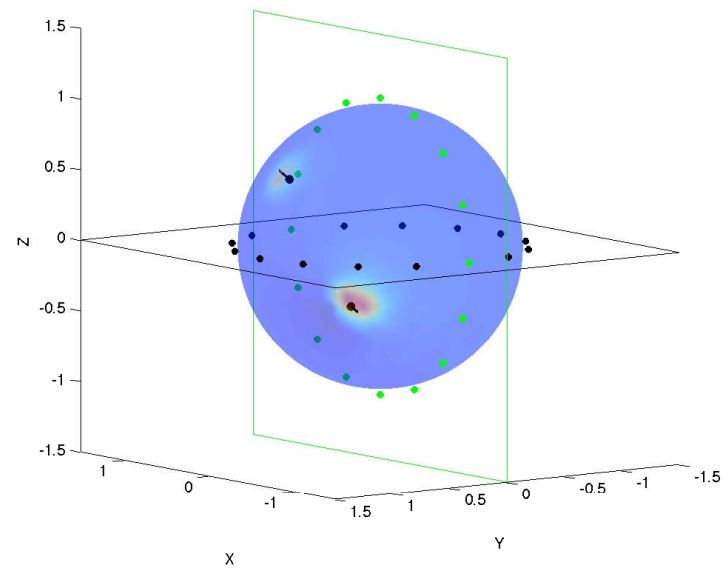

Fig. 1. Schematic view of the multi-planar analytic sensing approach. The domain of interest $\Omega$ is a homogeneous unit sphere. We show the rotation of the XY-plane around $X$ with angle $\theta$.

\subsection{Synthetic data}

We generate the electrical potential $V$ in 162 points on the surface $\partial \Omega$, using the forward model for two dipoles close to the surface:

$\mathbf{x}_{1}=\left[\begin{array}{l}0.8 \\ 0.2 \\ 0.4\end{array}\right], \mathbf{x}_{2}=\left[\begin{array}{c}-0.6 \\ 0.7 \\ -0.3\end{array}\right], \mathbf{p}_{1}=\left[\begin{array}{c}1 \\ 0 \\ 0.4\end{array}\right], \mathbf{p}_{2}=\left[\begin{array}{c}-2 \\ 1 \\ 0\end{array}\right]$

We deploy 16 analytic sensors (9) with $a_{n}=1.05 e^{i n \frac{\pi}{8}}$, $n=0, \cdots, 15$, in each of the 32 planes defined by $\theta_{m}=$ $\frac{\pi m}{32}, \phi_{m}=0, m=0, \ldots, 31$. As we only vary the $\theta$ angle, and the $x$-coordinate of the positions and moments are quite distinct, we are able to easily separate the dipoles for the 3-D recovery as suggested by (12).

To compute the surface integral, we make use of thin plate smoothing splines [3] without regularization. The measures are degraded by additive Gaussian noise at various SNR levels. In Fig. 1, we illustrate the general setup. Notice that with our approach, the two dipoles' positions are found at once as the roots of a quadratic equation, while methods based on numerical optimization using the forward model are prone to local minima due to the non-linear dependence on the positions.

In Fig. 2, we depict the sinograms for the various noise levels for the dipole positions (a) and dipole moments (b). Notice that for the noiseless case, we obtain perfect localization. As the noise level goes up, the estimates of the parameters gracefully degrade. It can be observed that the sinograms remain quite consistent. The relative errors with respect to the sphere's radius for the various SNR levels are listed in Table 1.

\begin{tabular}{c|c|c}
\hline \hline Parameter & relative error (10dB) & relative error (5dB) \\
\hline $\mathbf{x}_{1}$ & $0.2 \%$ & $4.6 \%$ \\
$\mathbf{p}_{1}$ & $3.6 \%$ & $12 \%$ \\
$\mathbf{x}_{2}$ & $0.1 \%$ & $4 \%$ \\
$\mathbf{p}_{2}$ & $3.2 \%$ & $10 \%$ \\
\hline \hline
\end{tabular}

Table 1. Relative errors with respect to the sphere's radius of the estimated dipoles' positions and moments at different noise levels.

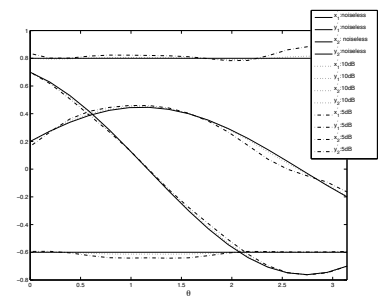

(a)

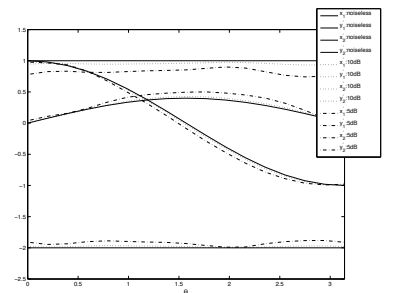

(b)
Fig. 2. Sinograms obtained for synthetic data at various SNR levels. (a) Dipole positions. The X'Y'-plane is obtained by rotation over the angle $\theta$ only. (b) Dipole moments.

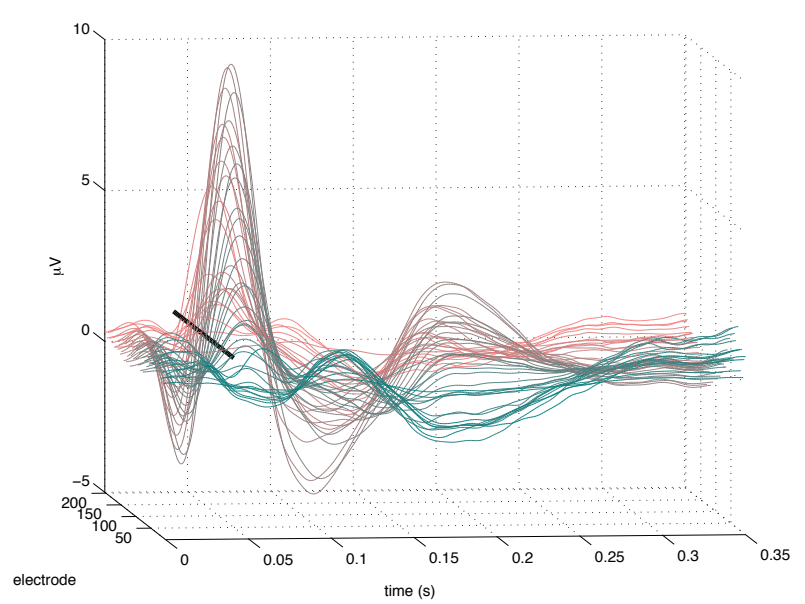

Fig. 3. The epoch-averaged EEG signal. The time point for fitting was chosen at the rise of the main positive peak (41ms post-stimulus). For visualization purposes, we only show time-courses for one out of four electrodes. 


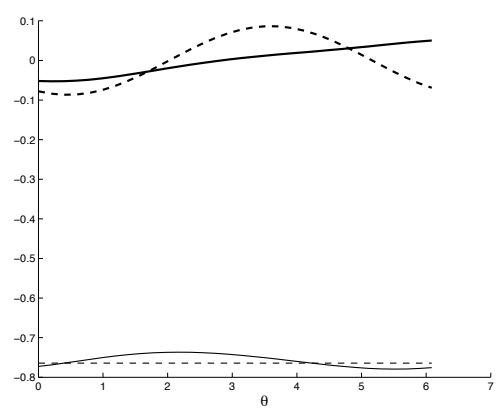

Fig. 4. Sinogram of the dipole's position obtained for experimental data. Full line: experimental sinogram; dashed line: fitted sinograms.

\subsection{Experimental Data}

We consider EEG data from visual ERPs (200 flashes to the left eye; epoch length $0.5 \mathrm{~s}$; sampling at $1 \mathrm{kHz} ; 204$ channels). The epoch-averaged signal is used for localization at the rise of the main positive peak (see Fig. 3). At this instant, the inverse solution using a reference method with distributed source model and the local autoregressive average (LAURA) in combination with SMAC (multi-layer spherical head model) [6] is most focalized ${ }^{1}$. The solution space for the distributed source model contains 3005 points uniformly distributed over the gray matter of the brain (mapped into the SMAC space), and their moments are oriented perpendicular to the cortical surface.

We deploy 32 analytic sensors in the same 32 planes as before. In Fig. 4, we depict the sinogram for a single dipole. Again, the estimation is consistent as the angle of the plane changes. In Fig. 5, we show the dipole intensities for the inverse solution using the distributed source model. A large activated region is reported in the occipital cortex. The fitted dipole using multi-planar analytic sensing is located at the border of the region detected by the refererence method. The reason that the dipole is less superficial is probably due to the fact that we do not consider the external layers (scalp, skull, cerebrospinal fluid) as in the SMAC model, which tends to localize more profoundly [7].

\section{CONCLUSION \& OUTLOOK}

In this paper, we demonstrated the feasibility of a multi-planar analytic sensing to EEG source localization. An attractive property of our method is that it provides a direct estimation even for multiple dipoles. While these results are promising, they also indicate a number of important issues that need to be addressed in future work.

- SMAC head model: Extend the analytic sensing ap-

\footnotetext{
${ }^{1}$ The CARTOOL software is available at http: //brainmapping. unige.ch/Cartool.htm.
}

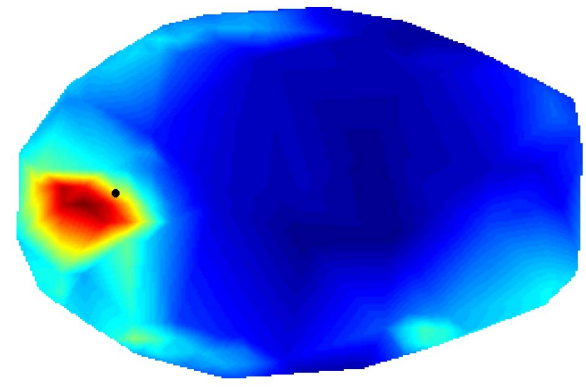

Fig. 5. The slice at $z=0.83 \mathrm{~cm}$ showing high intensities of the distributed source model as fitted by the reference method. The location of the dipole, $\mathbf{x}_{1}=\left[\begin{array}{llll}-0.76 & -0.010 .83\end{array}\right]^{T}$, found by multi-planar analytic sensing is superimposed (indicated by a black dot).

proach for a multishell model with piecewise constant conductivity.

- Interpolation method: Investigate the influence of the interpolation technique and its interaction with noise on the computing of the generalized measures.

- Alternative analytic sensors: Explore the use of other analytic sensors that could contribute to a more direct solution of the 3-D localization problem.

\section{REFERENCES}

[1] S. Baillet, J. C. Mosher, and R. M. Leahy. Electromagnetic brain mapping. IEEE Signal Processing Magazine, 18(6):1430, 2001

[2] D. Kandaswamy, T. Blu, and D. Van De Ville. Analytic sensing: Direct recovery of point sources from planar cauchy boundary measurements. Proceedings of the SPIE Optics 2007 Conference on Mathematical Methods: Wavelet XII, 6701, 2007.

[3] J. Kybic, T. Blu, and M. Unser. Generalized sampling: A variational approach part I: Applications. IEEE Transactions on Signal Processing, 50(8), 2003.

[4] C. M. Michel, M. M. Murray, G. Lantz, S. Gonzalez, L. Spinelli, and R. Grave de Peralta. EEG source imaging. Clin Neurophysiol, 115:2195-2222, 2004.

[5] Y. Salu, L. Cohen, D. Rose, S. Sato, C. Kufta, and M. Hallett. An improved method for localizing electric brain dipoles. IEEE Transactions on Biomedical Engineering, 37(7), 1990.

[6] L. Spinelli, S. G. Andino, G. Lantz, M. Seeck, and C. M. Michel. Electromagnetic inverse solutions in anatomically constrained spherical head models. Brain Topography, 13:115-125, 2000.

[7] C.J. Stok. The influence of model parameters on EEG/MEG single dipole source estimation. IEEE Transactions on Biomedical Engineering, 34(4):290-296, 1987.

[8] M. Vetterli, P. Marzilliano, and T. Blu. Sampling signals with finite rate of innovation. IEEE Transactions on Signal Processing, 50(6):1417-1428, 2002. 